Original Research

\title{
Curcumin ameliorates lipid metabolic disorder and cognitive dysfunction via the $A B C A 1$ transmembrane transport system in APP/PS1 double transgenic mice
}

\author{
Mingyuan Tian ${ }^{1,2, \dagger}$, Fanlin Zhou ${ }^{2,3, \dagger}$, Zhipeng Teng ${ }^{2}$, Chen Wang ${ }^{2}$, Xiong Zhang ${ }^{2}$, Yangyang Wang ${ }^{3}$, Yu Li $^{2,3, *}$ \\ ${ }^{1}$ Department of Endocrinology, The Second Affiliated Hospital of Chongging Medical University, 400010 Chongging, China \\ ${ }^{2}$ Institute of Neuroscience, School of Basic Medicine, Chongqing Medical University, 400016 Chongqing, China \\ ${ }^{3}$ Department of Pathology, Chongging University Cancer Hospital \& Chongqing Cancer Institute \& Chongqing Cancer Hospital, 400030 Chongqing, China \\ *Correspondence: liyu10o@cqu.edu.cn (Yu Li) \\ ${ }^{\dagger}$ These authors contributed equally.
}

DOI:10.31083/j.jin2004091

This is an open access article under the CC BY 4.0 license (https://creativecommons.org/licenses/by/4.0/).

Submitted: 14 September 2021 Revised: 9 October 2021 Accepted: 1 November 2021 Published: 30 December 2021

The disorder of lipid metabolism, especially cholesterol metabolism, can promote Alzheimer's Disease. Curcumin can ameliorate lipid metabolic disorder in the brain of Alzheimer's Disease patients, while the mechanism is not clear. APP/PS1 (APPswe/PSEN1dE9) double transgenic mice were divided into dementia, low-dose, and highdose groups and then fed for six months with different dietary concentrations of curcumin. Morris water maze was used to evaluate the transgenic mice's special cognitive and memory ability in each group. In contrast, the cholesterol oxidase-colorimetric method was used to measure total serum cholesterol and high-density lipoprotein levels. Immunohistochemistry was used to evaluate the expression of liver $X$ receptor $-\beta$, ATP binding cassette $A 1$ and apolipoprotein $A 1$ of the hippocampus and $A \beta 42$ in the brains of transgenic mice. The mRNA and protein expression levels of liver $X$ receptor- $\beta$, retinoid $X$ receptor- $a$ and ATP binding cassette $A 1$ were evaluated using qRTPCR and Western blotting, respectively. Curcumin improved the special cognitive and memory ability of transgenic Alzheimer's Disease Mice. The total serum cholesterol decreased in Alzheimer's Disease mice fed the curcumin diet, while the high-density lipoprotein increased. The curcumin diet was associated with reduced expression of $A \beta$ and increased expression of liver $X$ receptor- $\beta$, ATP binding cassette $A 1$, and apolipoprotein $A 1$ in the $C A 1$ region of the hippocampus. The mRNA and protein levels of retinoid $X$ receptor- $a$, liver $X$ receptor- $\beta$, and ATP binding cassette A1 were higher in the brains of Alzheimer's Disease mice fed the curcumin diet. Our results point to the mechanism by which curcumin improves lipid metabolic disorders in Alzheimer's Disease via the ATP binding cassette A1 transmembrane transport system.

\section{Keywords}

Curcumin; Alzheimer's disease; ATP binding cassette A1; Cholesterol metabolism; Transmembrane transport system

\section{Introduction}

Alzheimer's disease (AD) is an insidious neurodegenerative disorder. The clinical features of $\mathrm{AD}$ are progressive cognitive dysfunction and language and behavioral disorders, social dysfunction, and eventually death [1]. Cholesterol is an essential part of cell membranes and myelinated axons, thereby playing an essential role in maintaining neuronal integrity and functionality [2]. Although the brain is the organ with the highest cholesterol content in humans [3], it cannot absorb cholesterol directly from the blood because of the blood-brain barrier. Cholesterol synthesis in the brain is therefore carried out mainly by astrocytes and neurons [4] Cholesterol metabolism disorder is a major risk element to cardiovascular disease and may play a role in AD. Several clinical and epidemiological studies have reported a close link between cholesterol metabolism abnormality and AD [5].

ABCA1, a member of the ATP-binding cassette family, plays a pivotal role in cholesterol transport. The cholesterol transport regulated by ABCA1 also plays an essential part in the formation of $\beta$-amyloid $(\mathrm{A} \beta)$ deposits [6]. $\mathrm{ABCA} 1$ is a recently discovered important transmembrane protein that can mediate reverse cholesterol transport and promote the cellular efflux of cholesterol to various acceptors, including HDL (High-density lipoprotein) [7]. Under normal circumstances, the cholesterol transported by ABCA1 into the extracellular fluid can combine with apolipoprotein A1 (apoA1) to form $\beta$-HDL. $\beta$-HDL develops further into mature HDL, which can then help to reverse cholesterol transport [8]. ABCA1 in the body is mainly regulated by the liver $\mathrm{X}$ (LXR) and retinoid X (RXR) receptors. A recent study found the ABCA1 transmembrane transport system mediated by LXR and RXR played an essential role in cholesterol balance [9]. ABCA1 also participates in $\mathrm{A} \beta$ transport, deposition and senile plaque formation [2].

Curcumin is a special phenolic compound derived from the plant Curcuma longa. It is used clinically as an antioxidant, anti-inflammatory, anti-atherosclerosis, anticoagulation and anti-tumor agent [10]. The role of curcumin in reducing cholesterol levels is attracting more and more attention. Many researchers have started using cur- 
cumin to treat various transgenic animal models for diseases caused by lipid metabolism disorders, such as arteriosclerosis and hypercholesterolemia, with positive results. These studies demonstrated that curcumin could reduce cholesterol levels to maintain body balance $[11,12]$. However, the brain cholesterol level is not correlated with the blood cholesterol concentration, and its synthesis mechanism is also different [13]. It is unknown whether curcumin can reduce age-related changes in the brain and gradually increase cholesterol levels in the neurons of $\mathrm{AD}$ patients.

This research uses the APP/PS1 transgenic mouse model to observe the impact of different curcumin concentrations on cholesterol content and the LXR/RXR-mediated ABCA1 transmembrane transport system. This original experimental evidence should help to find new treatments and targets for AD.

\section{Materials and methods \\ 2.1 Animals}

APP/PSI (APPswe/PSEN1dE9) transgenic mice (Alzheimer's disease mouse model) and the type of wild C57BL/6J mice (control mice) were supplied through the Institute for Laboratory Animals, Nanjing Biomedical Research Institute, Nanjing University. Total animal experiments were carried out by the guidelines to Care and Use for experimental Laboratory Animals (NIH Publication No. 8523, revised 1996). They were accepted through the Research Ethics Committee of the Chongqing University Cancer Hospital. Six-month-old APP/PSI double transgenic mice were divided into three groups randomly. They fed different concentrations of curcumin-mixed feeds: the control group was fed a regular diet without curcumin. The Cur-L group was fed $0.16 \mathrm{~g} / \mathrm{kg}$ of curcumin- (Sigma-Aldrich) mixed, and Cur-H was fed $1.0 \mathrm{~g} / \mathrm{kg}$ of curcumin-mixed feeds. The experiments were carried out after feeding this diet for six months.

\subsection{Tissue preparation}

Mice were anesthetized by isoflurane deeply before obtaining tissue samples. A proportion of the mice was infused with $0.9 \%$ normal saline, and the brain tissues were stored at $-80{ }^{\circ} \mathrm{C}$. The remaining mice were infused with $0.9 \%$ normal saline containing $4 \%$ paraformaldehyde (PFA) solution.

\subsection{Antibodies}

Mouse anti-ABCA1 and goat anti-LXR- $\beta$ antibodies were purchased through Abcam. Rabbit anti-RXR- $\alpha$, mouse antiapoA1 and rabbit anti- $\beta$-actin were purchased through Santa Cruz. While secondary antibodies were purchased through Bioworld Technology.

\subsection{Morris water maze (MWM) test}

Specific protocols for the Morris water maze test were described in our previous research [14]. The experiment included a visual platform test (day 1 ), a hidden platform test (days 2 to 6), and a probe test (day 7).

\subsection{Measurement of serum total cholesterol (TC) and HDL}

Liquids were mixed according to instructions for the total cholesterol (CHO enzymatic) test kit and the serum highdensity lipoprotein kit (Nanjing Jiancheng Biological Engineering). Samples were then added according to the relevant instructions, and the absorbance was measured for each tube.

\subsection{Western blot assay}

The specific protocol for Western blot assay is described previously [14]. Protein from brain tissues was homogenized using a protein extraction reagent (RIPA:PMSF $=100: 1$ ). Protein concentrations were identified through the BCA assay (Beyotime, China). For Western blot analyses, proteins $(35 \mu \mathrm{g})$ were separated through SDS-PAGE (8\% or $10 \%$ gels) and then transferred to polyvinylidene fluoride (PVDF) membranes (Millipore, MS). After blocking, the membranes were incubated overnight at $4{ }^{\circ} \mathrm{C}$ with primary antibodies. After washing, the bound antibodies were detected by incubation for 1-2 $\mathrm{h}$ at room temperature with secondary antibodies. The membranes were then developed using a Commercial enhanced chemiluminescence system (ECL; Bio-rad), and quantified the signal using Quantity One image analysis (Bio-RAD, USA).

\subsection{Immunohistochemistry}

The brains of mice were fixed with $4 \%$ paraformaldehyde, embedded in paraffin, sliced five $\mu$ m thick, dewaxed with xylene and ethanol. After washing with TBS, Horseradish peroxidase (HRP) stained slides were treated with $0.3 \% \mathrm{H}_{2} \mathrm{O}_{2}$ for $30 \mathrm{~min}$ to inactivate endogenous peroxidase. Then the portions were blocked by $20 \%$ goat serum in TBS for one $h$. LXR- $\beta$, RXR- $\alpha$, ABCA, and apoA1 were detected with the corresponding antibodies, HRP-labeled avidin-biotin complex, and DAB substrate. Specimens were then observed using a fluorescence microscope (LEICA).

\subsection{The analysis of quantitative real-time PCR ( $q R T-P C R$ )}

RNA was separated through brain tissues using RNAiso Plus (TaKaRa). PrimeSriptTM RT reagent Kit (TaKaRa) compound first-strand cDNA based on the manufacturer's introduction. The cDNA was further amplified by SYBR Premix Ex TaqTM II (TaKaRa) in a $20 \mu \mathrm{L}$ reaction mix using the following primers: ABCA1-F: 5'-CAATGTCAAGGTGTGGTTCAAT-3'; ABCA1-R: $5^{\prime}$-GCTGCTGTTTAGTGAGGTTCAA- ${ }^{\prime}$; $\quad$ RXR- $\alpha$-F: $5^{\prime}$-TACGCAAAGACCTGACCTACAC- $3^{\prime}$; RXR- $\alpha-$ R: $5^{\prime}$-ACTCCACCTCGTTCTCATTCC- $3^{\prime} ; \quad$ LXR- $\beta-\mathrm{F}$; $5^{\prime}$-TAGAAACAGCCAGACGCTACAA- ${ }^{\prime}$; $\quad$ LXR- $\beta$-R: $5^{\prime}$-GGCGATAAGCAAGGCATACTCT- ${ }^{\prime} ; \quad \beta$-actin-F: $5^{\prime}$-TCCTGTGGCATCCACGAAACT- $3^{\prime} ; \quad \beta$-actin-R: 5'-GAAGCATTTGCGGTGGACGAT-3'.

\subsection{Statistical analysis}

Statistical analyses were performed using SPSS software (version 17.0, IBM Corp., Chicago, IL, USA). Experimental data are represented by the mean and standard error of the mean (SEM). Experimental results were compared using 

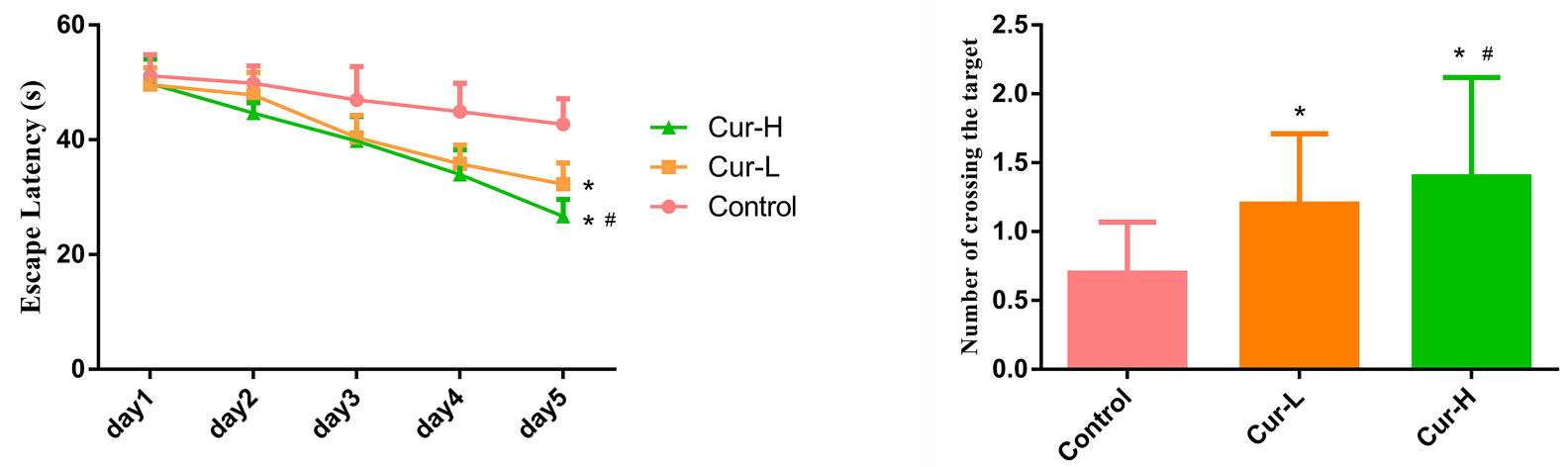

$\mathrm{C}$
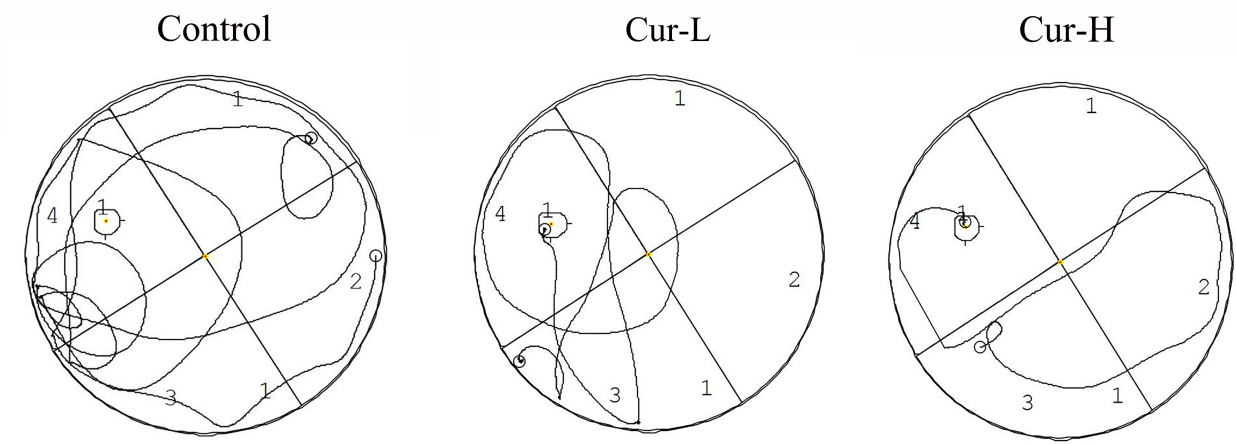

Fig. 1. Curcumin improved cognitive function of APP/PS1 double transgenic mice. (A) The escape latency during the 5-day MWM testes. (B) The number of crossing the target on the last day of the MWM test $\left(\mathrm{n}=5\right.$, two-way repeated-measures ANOVA, Tukey's test, $\# p<0.05$, versus Cur-L; ${ }^{*} p<0.01$, versus Control). (C) The representative route track map for every group to mice.

two-way ANOVA, next by multiple comparisons using Bonferroni post-test. $p<0.05$ was considered to show statistical significance.

\section{Results \\ 3.1 Curcumin improved cognitive function of APP/PS1 mice}

MWM was used to evaluate the hippocampal-dependent spatial learning and memory ability of the APP/PS1 double transgenic mouse model to AD. During the learning phase (Fig. 1A), the mice fed low-dose Curcumin (Cur-L group) demonstrated significantly shorter times to discover the evacuation platform (shorter escape latencies) compared to mice from the control group $(p<0.01)$. The average escape latency was also shortened in mice fed high-dose Curcumin (Cur-H group) $(p<0.01)$. In the probe test of spatial memory retention (Fig. 1B), the number of passes through the previous platform location was significantly reduced in the CUR-L group compared to the control group. The platform crossing frequency decreased even further in mice fed the higher curcumin dose $(p<0.01)$. Representative swim paths are displayed in Fig. 1C.

3.2 Curcumin reduced $A \beta$ in the hippocampus of APP/PS1 mice

Immunohistochemistry was used to detect $\mathrm{A} \beta$ plaque in APP/PS1 mice (Fig. 2). These plaques were extensive, mostly round, radiating, with a deep core in the control group. The fraction of occupied area and the number of amyloid protein plaques was lower in the Cur-L group (both $p<0.05$ ) than controls, and even more so in mice fed high-dose curcumin (both $p<0.01$ ).

\subsection{Curcumin reduced the level of TC and increased the level of HDL in the serum of APP/PS1 mice}

Using enzymatic detection, the serum TC levels for each group were found to be different (Fig. 3). Serum TC levels in the Cur-L group were dramatically lower than in the control group $(p<0.05)$, even more so in the Cur-H group ( $p<$ $0.05)$. However, there was no difference significantly in the serum TC level between the low- and high-dose curcumin groups $(p>0.05)$. Colorimetric detection showed the serum HDL levels of each group were also notably different (Fig. 3); The serum HDL levels in both the Cur-L and Cur-H groups were significantly higher than those in the control group ( $p$ $<0.05)$.

\subsection{Curcumin promoted the expression of $A B C A 1$ and apoA1 in the hippocampus of APP/PS1 mice}

Western blotting and immunocytochemistry were used to detect the ABCA1 expression and apoA1 in each group. Lowdose curcumin treatment enhanced the expression of ABCA1 and apoA1 in the hippocampus of APP/PS1 double trans- 
A

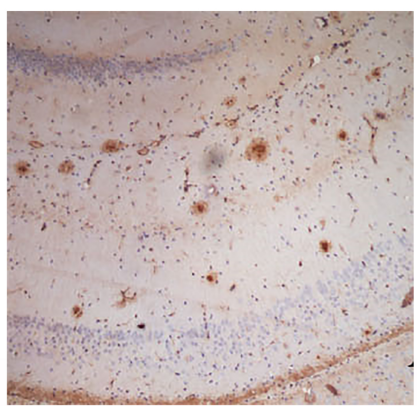

B

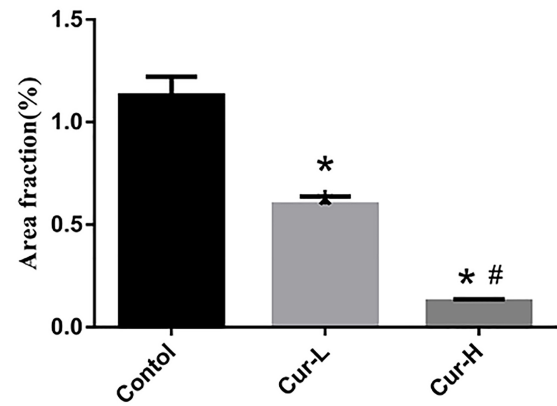

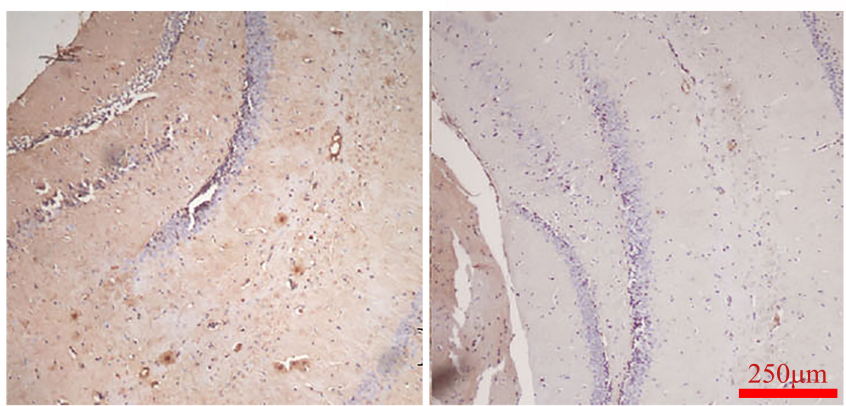

$\mathrm{C}$

Fig. 2. Curcumin reduced $\mathbf{A} \beta$ in the hippocampus of APP/PS1 mice. (A) Immunohistochemical staining displays amyloid protein was expressed in the hippocampus of different groups (Scale bar $=250 \mu \mathrm{m})$. (B,C) Area fraction and number of plaques of $\mathrm{A} \beta$ in every group (\#p<0.05, versus Cur-L; ${ }^{*} p<0.01$, versus Control).

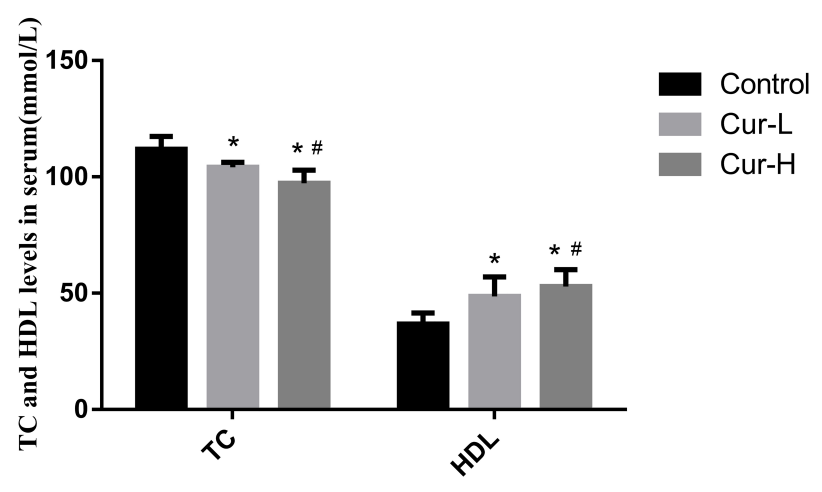

Fig. 3. Curcumin reduced the level of TC and increased HDL in the serum of APP/PS1 mice $(\# p>0.05$, versus Cur-L; * $p<0.05$, versus Control).

genic mice (both $p<0.05$ ). High-dose Curcumin promoted the expression even more, as indicated by the increased intensity of immunocytochemical staining (Fig. 4).

\subsection{Curcumin promoted the expression of RXR- $\alpha$ and $L X R-\beta$ in the hippocampus of APP/PS1 mice}

Western blotting, qRT-PCR and immunocytochemistry were used to evaluate the expression of RXR- $\alpha$ and LXR$\beta$ in each group. The expression of $\mathrm{RXR}-\alpha$ and LXR- $\beta$ at the mRNA standard was significantly increased in Cur-L and Cur-H groups compared with the control group (both $p<0.05$ ) (Fig. 5A,B). Low-dose curcumin treatment increased the expression of RXR- $\alpha$ and LXR- $\beta$ proteins in the hippocampus of APP/PS1 double transgenic mice (both $p<0.05)$. High-dose Curcumin increased the expression of RXR- $\alpha$ and LXR- $\beta$ protein even more, as indicated by the increased intensity of immunocytochemical staining (Fig. 5CI).

\section{Discussion}

In neuroscience, it is assumed that the critical pathological change in $\mathrm{AD}$ is the deposition of $\mathrm{A} \beta[15,16]$. Our earlier research showed that cognitive function was damaged and insoluble $\mathrm{A} \beta$ deposition gradually enhanced with age in the hippocampus of APP/PS1 mice [14]. Therefore, discovering a compound that reduces both $\mathrm{A} \beta$ deposition and cognitive impairment offers new hope for $\mathrm{AD}$ treatment. Curcumin is the main component of turmeric, a commonly used Chinese medicine derived from a plant compound. Many in vitro and in vivo experiments have shown that curcumin has anti-inflammatory, antioxidant, and anti-amyloid deposition properties [17]. Earlier studies found that curcumin reduces amyloid plaques by directly binding to and disaggregating amyloid and inhibiting the aggregation of $\mathrm{A} \beta$ [18]. Previous in vitro studies by our group found that curcumin can in- 
A

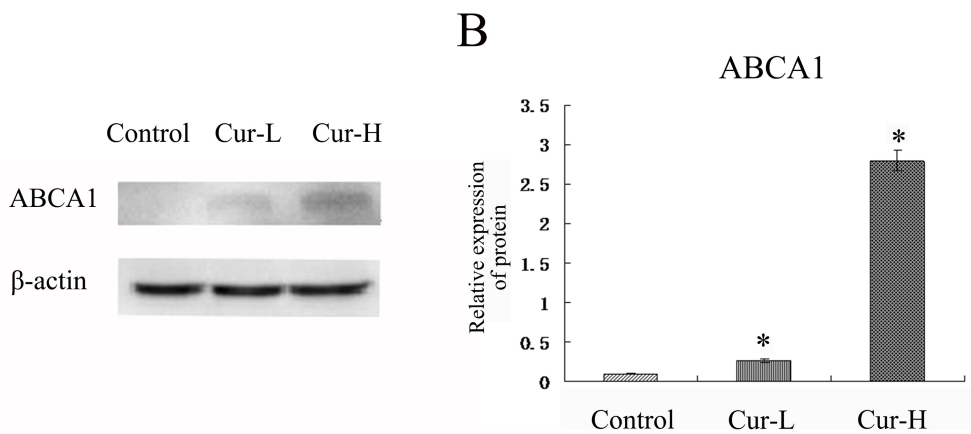

C

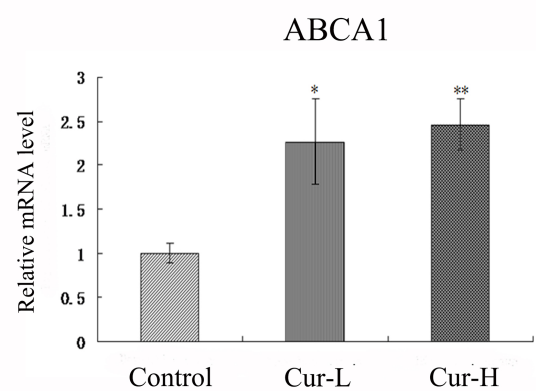

D

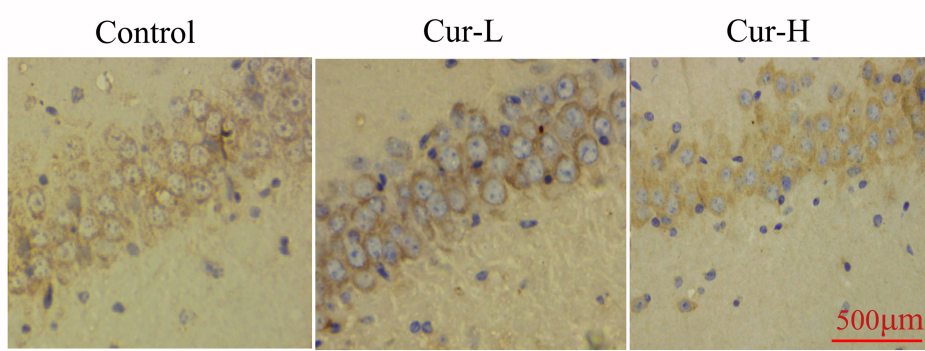

F

Control

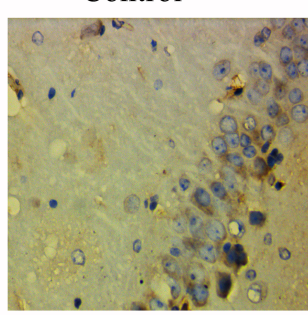

Cur-L

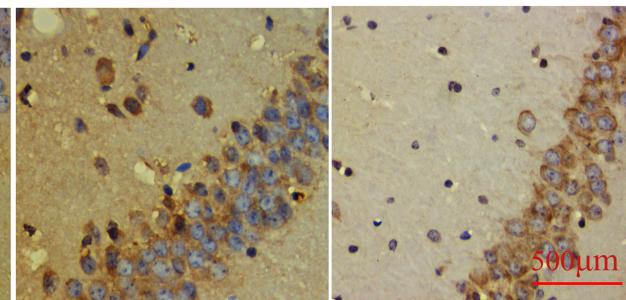

E

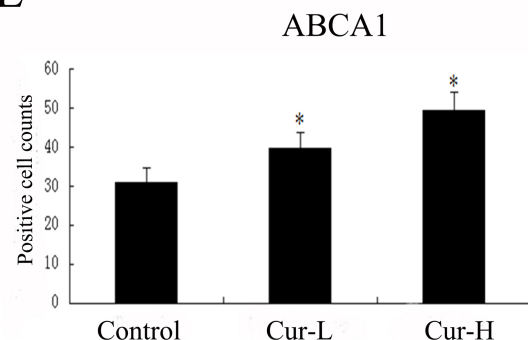

G

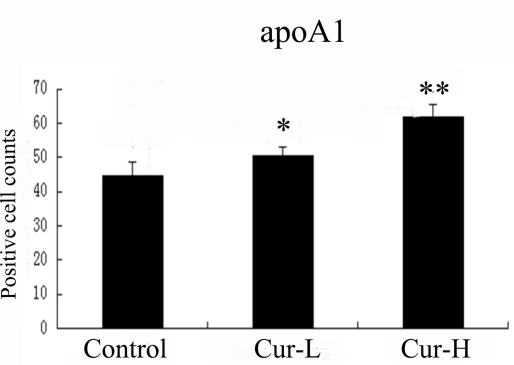

Fig. 4. Curcumin promoted the ABCA1 expression and apoA1 in the hippocampus of APP/PS1 mice. (A-C) Western blot and RT-PCR analysis to ABCA1 of each group. (D-G) Immunocytochemistry analysis of ABCA1 and apoA1 in each group (Scale bar $=500 \mu \mathrm{m}$ ). The data are mean \pm SEM of a typical three experimental series $\left({ }^{*} p<0.05\right.$, versus Control; ${ }^{* *} p<0.01$, versus Control).

hibit the production of $\mathrm{A} \beta$ via the $\mathrm{PI} 3 \mathrm{~K} / \mathrm{Akt} / \mathrm{mTOR}$ signaling pathway [19]. Therefore, many studies have shown that curcumin regulates the formation and aggregation of $\mathrm{A} \beta$, but the exact mechanism remains unclear.

To further explore the protective effects of Curcumin against $\mathrm{AD}$, the MWM test suggested that curcumin improved cognitive function in the hippocampus of APP/PS1 mice. Curcumin also reduced $\mathrm{A} \beta$ deposition in the hippocampus for mice. This result was consistent with our previous in vitro experiments [17].

Recent work suggests that AD is associated with cholesterol metabolism disorder. Cholesterol is an essential part of cell membranes and myelinated axons and plays an essential role in keeping the integrity and functionality of neurons [2]. However, the brain cannot obtain cholesterol from the blood due to the blood-brain barrier [4]. Synthesis by astrocytes and neurons is the primary source of cholesterol in brain tissues [20]. Epidemiological studies have found that patients with hypercholesterolemia were more likely to suf- fer from $\mathrm{AD}$ and that taking cholesterol-lowering drugs could reduce the incidence of $\mathrm{AD}[21,22]$. Transgenic mice with hyper cholesterol and rabbits on a high-cholesterol diet both showed cholesterol metabolism disorder that could promote amyloid deposition and lead to AD and cognitive dysfunction $[23,24]$. The total cholesterol (TC) and low-density lipoprotein (LDL) levels of AD patients were higher than those of ordinary people [25]. These studies suggested an association between $\mathrm{AD}$ and cholesterol metabolism disorder. In the present research, curcumin reduced the TC level in APP/PS1 mice serum, with high-dose curcumin being more effective than low-dose curcumin. This finding is similar to Shin et al. [26], who found that curcumin significantly lowered the cholesterol level.

The ABCA1 transmembrane system is a one-way cholesterol transporter that mediates outward transport after conjugating free cholesterol and phospholipid. ABCA1 is a member of the ATP-binding cassette family [27]. Structurally, it has two transmembrane segments and two nucleotide- 
A

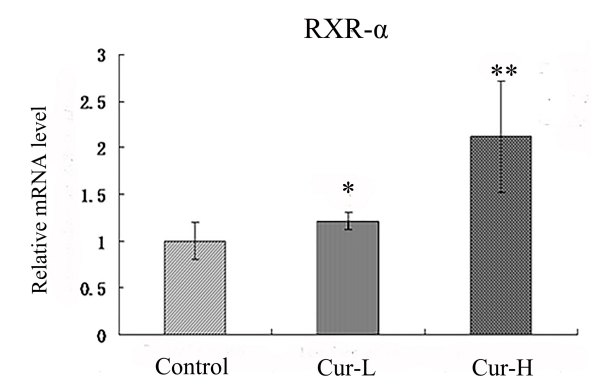

C

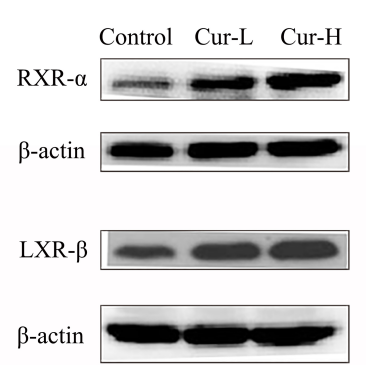

$\mathrm{D}$

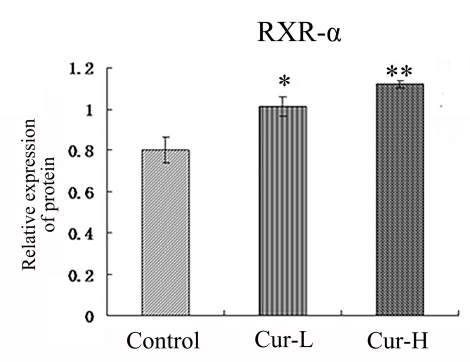

F

Control

Cur-L

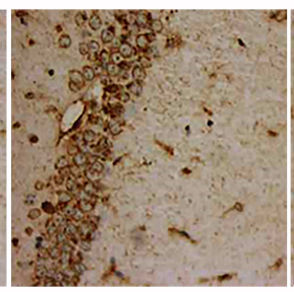

H

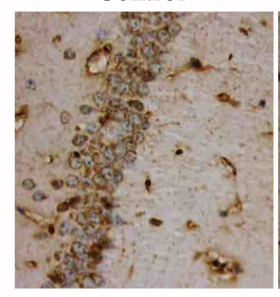

Control

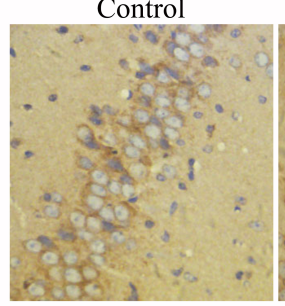

B

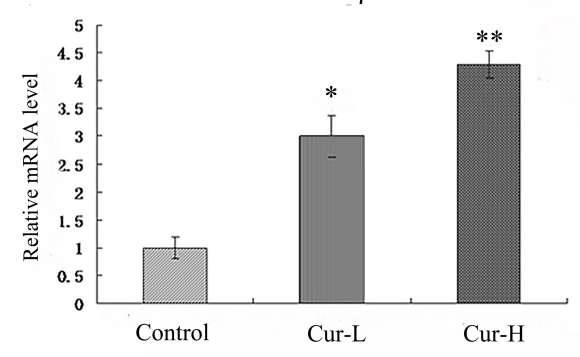

E

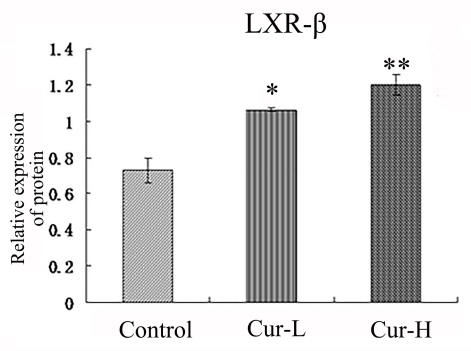

G
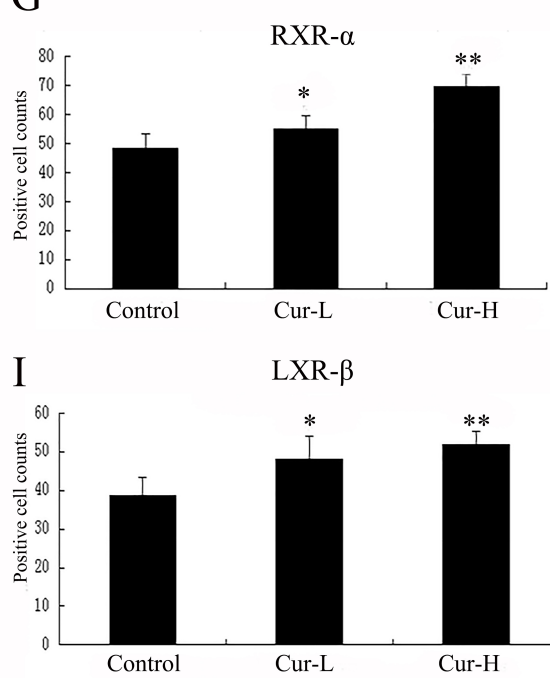

Fig. 5. Curcumin promoted the expression of RXR- $\alpha$ and LXR- $\beta$ in the hippocampus of APP/PS1 mice. (A,B) Relative mRNA expression of RXR- $\alpha$ and LXR- $\beta$ of every group. (C-E) Western blot analysis of RXR- $\alpha$ and LXR- $\beta$ in each group. (F-I) Immunocytochemistry analysis of RXR- $\alpha$ and LXR- $\beta$ in each group (Scale bar $=500 \mu \mathrm{m})$. The data stands for as mean \pm SEM of a typical three experimental series $\left({ }^{*} p<0.05\right.$, versus Control; ${ }^{* *} p<0.01$, versus Control).

binding domains. The nucleotide-binding site can bind ATP to provide sufficient energy to support the transmembrane transport of cholesterol. This way, ABCA1 substrates such as free cholesterol and phospholipid can be transferred to the cell exterior [28]. ABCA1 is mainly regulated by LXR and $\mathrm{RXR}$. The former is a nuclear receptor for oxidized cholesterol, a member of the nuclear receptor superfamily, and a store of cholesterol content in many kinds of cells. LXR has two subtypes: LXR- $\alpha$ and LXR- $\beta$. LXR- $\beta$ is widely distributed in the body and is highly expressed in the brain (2 5 times more than in the liver) $[27,29]$. An increased cholesterol level in the brain can activate the LXR- $\beta$ lig- and, which in turn activates LXR- $\beta$. Activated LXR- $\beta$ can form heterodimers with RXR, which then combine with specific DNA components (LXRE) to alter the expression of target genes such as ABCA1 [30]. RXR owns three subtypes, namely $\mathrm{RXR}-\alpha$, RXR $-\beta$, and $\mathrm{RXR}-\gamma$. The former is mainly expressed in the hippocampus and neocortex, while the latter is only found in the neocortex [31]. Recent studies have shown that the LXR and ABCA1 cholesterol transport systems are essential in balancing nerve cells' interior and exterior cholesterol levels [32]. Moreover, the occurrence and development of AD were also closely related to this system. 


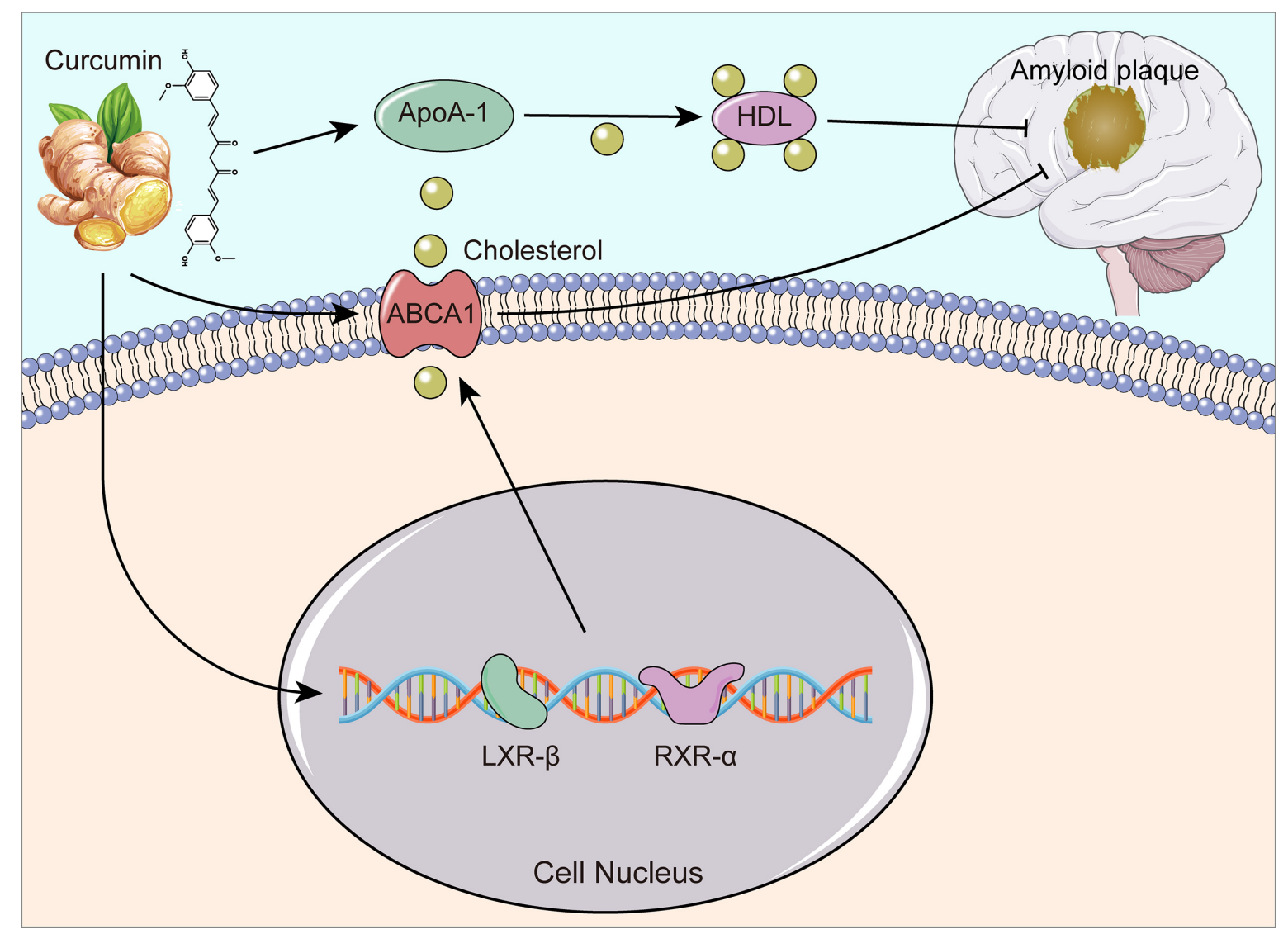

Fig. 6. Schematic diagram about the curcumin's possible mechanisms. Curcumin could promote the expression of LXR- $\beta$ and RXR- $\alpha$, which activated the ABCA1 cholesterol transmembrane system, thus maintaining the metabolic balance between intracellular and extracellular cholesterol. At the same time, curcumin reduces the serum total cholesterol level by increasing HDL levels and its source protein apoA1. In addition, the effect of curcumin on inhibiting the deposition of $\mathrm{A} \beta$ in the brain of mice may be achieved through the pathways mentioned above. Therefore, curcumin can improve lipid metabolic disorder and cognitive dysfunction by promoting the ABCA1 transmembrane transport system.

Jiang et al. [33] found that amyloid deposition in the brain of aging AD model mice (Tg2576) was dramatically decreased following treatment with GW3965, an agonist of LXR. At the same time, the memory capacity of these transgenic mice showed improvement. Fitz et al. [34] confirmed that treatment with LXR agonist could reduce amyloid deposition in the brains of transgenic mice fed a high-fat diet, as well as improve their cognitive function. Fitz et al. [34] also reported that LXR agonists could promote the activation of LXR- $\alpha /$ LXR- $\beta$ in vitro. This activated the ABCA 1 cholesterol transmembrane system, thus maintaining the metabolic balance between intracellular and extracellular cholesterol.

We observed increased expression of RXR- $\alpha$ and LXR$\beta$ at the protein and mRNA levels in the hippocampus of APP/PS1 mice fed curcumin for six months. Feeding with a higher dose of curcumin led to an even more significant increase. The production of $\mathrm{A} \beta$ decreased markedly after curcumin feeding, accompanied by increased LXR and ABCA1 expression. The ABCA1 cholesterol transmembrane transport system, therefore, plays a significant part in maintaining the balance of intracellular and extracellular cholesterol and participates in the transport and deposition of $\mathrm{A} \beta$ and the formation of senile plaques. Hence, it could become a novel target for the treatment of AD. We also propose for the first time that curcumin is a natural agonist which increases the LXR- $\beta$ and RXR- $\alpha$ expression. These can then be combined to promote the expression of ABCA1.

Following its transfer by ABCA1 to the extracellular fluid, cholesterol can combine with ApoA1 bound to the cell surface to form HDL. This carries cholesterol to the liver and reduces the cholesterol level due to the production of bile [34]. The generally accepted view is that increased HDL levels and its source protein apoA1 can lessen the probability of cardiovascular disease. Mature HDL is the smallest spherical particle amongst plasma lipoproteins and can scavenge intravascular lipid. Low HDL levels can lead to aberrant cholesterol metabolism and lipid deposition [35]. Recent clinical studies have shown that HDL and apoA1 levels in the plasma of $\mathrm{AD}$ patients are notably reduced [36]. Lewis et al. [37] previously reported the HDL level in APP/PS1/apoA1 transgenic mice was double that observed in APP/PS1 transgenic mice. These workers used the water maze test to confirm that APP/PS1/apoA1 transgenic mice did not appear to have agerelated learning and memory deficits, in direct contrast to the 
results for APP/PS1 transgenic mice. Lewis et al. [37] further suggested that plasma HDL and apoA1 levels were closely related to the cognitive function of $\mathrm{AD}$ patients. Our results also indicate that HDL has a protective effect against $A D$, since the HDL level increased as the level of $\mathrm{A} \beta$ decreased. Furthermore, the apoA1 expressed in the CA1 region to the hippocampus increased significantly in curcumin-fed mice, especially in the high-dose group. This observation is also consistent with the other findings mentioned above. The cholesterol carried by HDL and apoA1 is transferred from the cell interior by ABCA1.

\section{Conclusions}

Our results show that curcumin can improve lipid metabolic disorder and cognitive dysfunction by promoting the ABCA1 transmembrane transport system (Fig. 6). Furthermore, curcumin may become an ideal medication to prevent and treat $\mathrm{AD}$ due to its multitarget activity as a natural agonist in the ABCA1 transmembrane transport system. One outstanding question that warrants further study is whether the ABCA1 transmembrane transport system is the most important mechanism by which curcumin can ameliorate $\mathrm{AD}$ symptoms. Therefore, we will continue to explore the impacts of curcumin on the ABCA1 transmembrane transport system and lipid metabolism in vivo, and it offers new ideas for the treatment of AD.

Though there is essential evidence that curcumin may act on multiple pathways identified in AD's pathogenesis, its low bioavailability and low blood-brain barrier (BBB) penetration limit the role of Curcumin in $\mathrm{AD}$ [38], studies exploring nanoparticle technology found that liposomal curcumin, polymeric nano curcumin and poly-lactic-co-glycolic acid copolymer-curcumin could increase oral bioavailability or cross the BBB $[39,40]$. Therefore, improving the bioavailability, $\mathrm{BBB}$ penetration and maintaining the half-life of curcumin is also a primary future goal.

\section{Abbreviations}

$\mathrm{AD}$, Alzheimer's disease; ABCA1, ATP-binding cassette transporter A1; $\mathrm{A} \beta, \beta$-amyloid; apoA1, apolipoprotein $\mathrm{A} 1$; APP/PSI, APPswe/PSEN1dE9; Cur-H, High-dose curcumin; Cur-L, Low-dose curcumin; HDL, High-density lipoprotein; LXR, Liver X receptor; LDL, Low lipoprotein; MWM, The Morris water maze; RXR, Retinoid X receptor; TC, Total cholesterol.

\section{Author contributions}

YL, FLZ, XZ and MYT designed the experiments; MYT, FLZ, ZPT, XZ, YW and CW performed the experiments; FLZ, MYT, ZPT and YYW helped in data analysis and paper writing. All authors have read and approved the manuscript.

\section{Ethics approval and consent to participate}

Animals were raised and handled at the Chongqing University Cancer Hospital. All animal care protocols and exper- iments were reviewed and approved by the Ethics Committee of Chongqing University Cancer Hospital (CZLS2021244A). The consent for publication was obtained from all authors.

\section{Acknowledgment}

We thank two anonymous reviewers for their excellent criticism of the article.

\section{Funding}

This work was supported by the National Natural Science Foundation of China (NSFC: 81671261, 81801389) and the Natural Science Foundation of Chongqing (No. cstc2017jcyjAX0050).

\section{Conflict of interest}

The authors declare no conflict of interest.

\section{References}

[1] Wang YY, Yan Q, Huang ZT, Zou Q, Li J, Yuan MH, et al. Ameliorating Ribosylation-Induced Amyloid- $\beta$ Pathology by Berberine via Inhibiting mTOR/p70S6K Signaling. Journal of Alzheimer's Disease. 2021; 79: 833-844.

[2] Behl T, Kaur I, Sehgal A, Kumar A, Uddin MS and Bungau S. The Interplay of ABC Transporters in Abeta Translocation and Cholesterol Metabolism: Implicating Their Roles in Alzheimer's Disease. Molecular Neurobiology. 2021; 58:1564-1582.

[3] Dierckx T, Bogie JFJ, Hendriks JJA. The Impact of Phytosterols on the Healthy and Diseased Brain. Current Medicinal Chemistry. 2019; 26: 6750-6765.

[4] Pfrieger FW, Ungerer N. Cholesterol metabolism in neurons and astrocytes. Progress in Lipid Research. 2011; 50: 357-371.

[5] Samant NP, Gupta GL. Novel therapeutic strategies for Alzheimer's disease targeting brain cholesterol homeostasis. European Journal of Neuroscience. 2021; 53: 673-686.

[6] Wahrle SE, Jiang H, Parsadanian M, Kim J, Li A, Knoten A, et al. Overexpression of ABCA1 reduces amyloid deposition in the PDAPP mouse model of Alzheimer disease. Journal of Clinical Investigation. 2008; 118: 671-682.

[7] Yvan-Charvet L, Wang N, Tall AR. Role of HDL, ABCA1, and ABCG1 Transporters in Cholesterol Efflux and Immune Responses. Arteriosclerosis, Thrombosis, and Vascular Biology. 2010; 30: 139-143.

[8] Repa JJ, Li H, Frank-Cannon TC, Valasek MA, Turley SD, Tansey $\mathrm{MG}$, et al. Liver X Receptor Activation Enhances Cholesterol Loss from the Brain, Decreases Neuroinflammation, and Increases Survival of the NPC1 Mouse. Journal of Neuroscience. 2007; 27: 14470-14480.

[9] Gao MN and Li Y. The relationship between LXR/RXR and ABCA1 transmembrane-transport system with Alzheimer's disease. Progress in Physiological Sciences. 2011; 42: 460-462. (In Chinese)

[10] Aggarwal BB, Sung B. Pharmacological basis for the role of curcumin in chronic diseases: an age-old spice with modern targets. Trends in Pharmacological Sciences. 2009; 30: 85-94.

[11] Arafa HM. Curcumin attenuates diet-induced hypercholesterolemia in rats. Medical Science Monitor. 2005; 11: BR228BR2234.

[12] Peschel D, Koerting R, Nass N. Curcumin induces changes in expression of genes involved in cholesterol homeostasis. The Journal of Nutritional Biochemistry. 2007; 18: 113-119.

[13] Zhang J, Liu Q. Cholesterol metabolism and homeostasis in the brain. Protein \& Cell. 2015; 6: 254-264. 
[14] Zhou F, Xiong X, Li S, Liang J, Zhang X, Tian M, et al. Enhanced autophagic retrograde axonal transport by dynein intermediate chain upregulation improves $\mathrm{A} \beta$ clearance and cognitive function in APP/PS1 double transgenic mice. Aging (Albany NY). 2020; 12: 12142-12159.

[15] Zhu Y, Peng L, Hu J, Chen Y, Chen FX. Current anti-Alzheimer's disease effect of natural products and their principal targets. Journal of Integrative Neuroscience. 2019, 18: 327-339.

[16] Benilova I, Karran E and De Strooper B. The toxic A $\beta$ oligomer and Alzheimer's disease: an emperor in need of clothes. Nature Neuroscience. 2012; 15: 349-357.

[17] Ringman J, Frautschy S, Cole G, Masterman D, Cummings J. A Potential Role of the Curry Spice Curcumin in Alzheimers Disease. Current Alzheimer Research. 2005; 2: 131-136.

[18] Yang F, Lim GP, Begum AN, Ubeda OJ, Simmons MR, Ambegaokar SS, et al. Curcumin inhibits formation of amyloid beta oligomers and fibrils, binds plaques, and reduces amyloid in vivo. Journal of Biological Chemistry. 2005; 280: 5892-5901.

[19] Wang C, Zhang X, Teng Z, Zhang T, Li Y. Downregulation of PI3K/Akt/mTOR signaling pathway in curcumin-induced autophagy in APP/PS1 double transgenic mice. European Journal of Pharmacology. 2014; 740: 312-320.

[20] Chen W, Chen G, Head DL, Mangelsdorf DJ, Russell DW. Enzymatic reduction of oxysterols impairs LXR signaling in cultured cells and the livers of mice. Cell Metabolism. 2007; 5: 73-79.

[21] LaFerla FM and Oddo S. Alzheimer's disease: Abeta, tau and synaptic dysfunction. Trends in Molecular Medicine. 2005; 11: 170-176.

[22] Schuster GU, Johansson L, Kietz S, Stulnig TM, Parini P, Gustafsson J. Improved metabolic control by depletion of Liver X Receptors in mice. Biochemical and Biophysical Research Communications. 2006; 348: 176-182.

[23] Selkoe DJ. Alzheimer's disease: genes, proteins, and therapy. Physiological Reviews. 2001; 81: 741-766.

[24] Holsinger RMD, Goense N, Bohorquez J, Strappe P. Splice variants of the Alzheimer's disease beta-secretase, BACE1. Neurogenetics. 2013; 14: 1-9.

[25] Puglielli L, Tanzi RE, Kovacs DM. Alzheimer's disease: the cholesterol connection. Nature Neuroscience. 2003; 6: 345-351.

[26] Shin S, Ha T, McGregor RA, Choi M. Long-term curcumin administration protects against atherosclerosis via hepatic regulation of lipoprotein cholesterol metabolism. Molecular Nutrition and Food Research. 2011; 55: 1829-1840.

[27] Sano M, Bell KL, Galasko D, Galvin JE, Thomas RG, van Dyck $\mathrm{CH}$, et al. A randomized, double-blind, placebo-controlled trial of simvastatin to treat Alzheimer disease. Neurology. 2011; 77: 556563.

[28] Do TM, Ouellet M, Calon F, Chimini G, Chacun H, Farinotti R, et al. Direct evidence of abca1-mediated efflux of cholesterol at the mouse blood-brain barrier. Molecular and Cellular Biochemistry. 2011; 357: 397-404.
[29] Zambón D, Quintana M, Mata P, Alonso R, Benavent J, CruzSánchez F, et al. Higher Incidence of Mild Cognitive Impairment in Familial Hypercholesterolemia. The American Journal of Medicine. 2010; 123: 267-274.

[30] Koldamova R, Fitz NF, Lefterov I. The role of ATP-binding cassette transporter A1 in Alzheimer's disease and neurodegeneration. Biochimica et Biophysica Acta - Biomembranes. 2010; 1801: 824-830.

[31] Akanuma S, Hori S, Ohtsuki S, Fujiyoshi M, Terasaki T. Expression of nuclear receptor mRNA and liver $\mathrm{X}$ receptor-mediated regulation of $\mathrm{ABC}$ transporter $\mathrm{A} 1$ at rat blood-brain barrier. Neurochemistry International. 2008; 52: 669-674.

[32] Saint-Pol J, Vandenhaute E, Boucau MC, Candela P, Dehouck $\mathrm{L}$, Cecchelli $\mathrm{R}$, et al. Brain pericytes ABCA1 expression mediates cholesterol efflux but not cellular amyloid- $\beta$ peptide accumulation. Journal of Alzheimer's Disease. 2012; 30: 489-503.

[33] Jiang Q, Lee CYD, Mandrekar S, Wilkinson B, Cramer P, Zelcer $\mathrm{N}$, et al. ApoE Promotes the Proteolytic Degradation of A $\beta$. Neuron. 2008; 58: 681-693.

[34] Soumian S, Albrecht C, Davies A, Gibbs R. ABCA1 and atherosclerosis. Vascular Medicine. 2005; 10: 109-119.

[35] Tsompanidi EM, Brinkmeier MS, Fotiadou EH, Giakoumi SM, Kypreos KE. HDL biogenesis and functions: role of HDL quality and quantity in atherosclerosis. Atherosclerosis. 2010; 208: 3-9.

[36] Höglund K, Thelen KM, Syversen S, Sjögren M, von Bergmann $\mathrm{K}, \mathrm{W}$ allin $\mathrm{A}$, et al. The effect of simvastatin treatment on the amyloid precursor protein and brain cholesterol metabolism in patients with Alzheimer's disease. Dementia and Geriatric Cognitive Disorders. 2005; 19: 256-265.

[37] Lewis TL, Cao D, Lu H, Mans RA, Su YR, Jungbauer L, et al. Overexpression of human apolipoprotein A-I preserves cognitive function and attenuates neuroinflammation and cerebral amyloid angiopathy in a mouse model of Alzheimer disease. Journal of Biological Chemistry. 2010; 285: 36958-36968.

[38] Goozee KG, Shah TM, Sohrabi HR, Rainey-Smith SR, Brown B, Verdile G, et al. Examining the potential clinical value of curcumin in the prevention and diagnosis of Alzheimer's disease. British Journal of Nutrition. 2016; 115: 449-465.

[39] Shaikh J, Ankola DD, Beniwal V, Singh D, Kumar MNVR. Nanoparticle encapsulation improves oral bioavailability of curcumin by at least 9 -fold when compared to curcumin administered with piperine as absorption enhancer. European Journal of Pharmaceutical Sciences. 2009; 37: 223-230.

[40] Chiu SS, Lui E, Majeed M, Vishwanatha JK, Ranjan AP, Maitra A, et al. Differential distribution of intravenous curcumin formulations in the rat brain. Anticancer Research. 2011;31: 907-911. 